\title{
Airway macrophage carbon: biomarker of exposure to particulate matter air pollution
}

\author{
ERS Annual Award for Paediatric Respiratory Research in Europe, sponsored by AstraZeneca
}

\section{Neeta Kulkarni}

Dept of Infection, Immunity and inflammation, University of Leicester, Leicester, UK

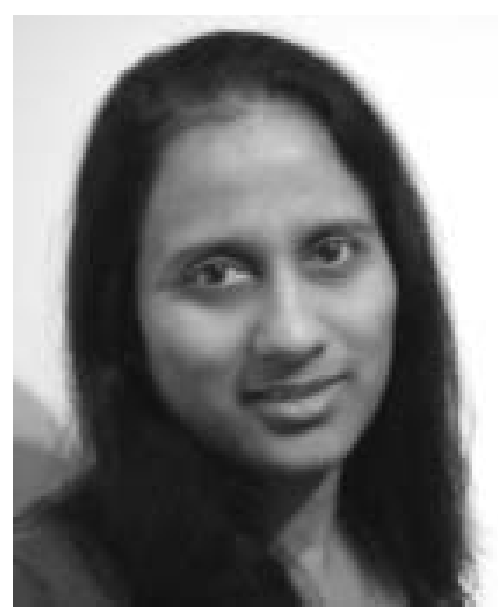

\section{MY JOB AND UNIT IN WHICH I WORK}

The research presented here was conducted at the Division of Child Health at the University of Leicester (Leicester, UK). I was a research fellow on the project supervised by Professor Jonathan Grigg. Professor Grigg (who has been presented with the Occupational and Environmental Best Paper Annual Award 2007 by the European Respiratory Society) is currently based at Queen Mary University of London (London, UK), and I am now collaborating with him on future research. The University of Leicester has links with the Gondar Institute of Medical Sciences in Ethiopia (the Leicester-Gondar Link) for sharing skills and expertise between health professionals. We are also fortunate to have a cohort group, established by Professor M. Silverman and Dr C. Kuehni, which follows children with wheeze and asthma. One of the research interests of the group is to assess associations of traffic proximity and respiratory symptoms in cohort children. Professor Chris O'Callaghan (Professor of Paediatrics and Head of the Child Health Division at the University of Leicester) leads the National Referral Service for investigation of patients with primary ciliary dyskinesia. His research group is investigating the effect of bacteria and viruses on the human ciliated respiratory epithelium.

I am also collaborating with Professors Christopher Brightling and Andrew Wardlaw at the Institute for Lung Health, University

STATEMENT OF INTEREST: None declared. of Leicester, and Professor Peter Barnes at Imperial College London to study the mechanisms of impaired particle uptake by airway macrophages (AM) in asthma. The main research interest of Professor Wardlaw is eosinophil trafficking and activation in asthma, and that of Prof. Brightling is to understand the interactions between mast cells and airway smooth muscle cells in the development of the asthmatic phenotype. I collaborated with Dr V.D. Patil at the Jawaharlal Nehru Medical College, Belgaum, India, to investigate health effects of biomass smoke exposure in children.

\section{A SYNOPSIS OF MY RESEARCH}

Epidemiological studies indicate that particulate matter (PM) from combustion of fossil and biomass fuels is associated with adverse health effects in children and adults. One of the limitations of the existing epidemiological studies is the assessment of individual lower airway exposure to PM, a site more relevant for adverse health effects. Since carbon is a major chemical component of traffic sources of PM and as AM ingest inhaled particles, we used AM carbon content as a measure of airway exposure to correlate with adverse health effects.

Children are exposed to a much higher level of PM in developing countries, from the burning of biomass fuels, such as wood, animal dung and crop residues. These fuels are used for cooking, heating and boiling water. This widespread exposure to PM from biomass smoke is estimated to cause two million excess deaths per year, a major proportion of these being in young children. It increases the risk of acute respiratory infections, otitis media and tuberculosis in children, and also chronic obstructive lung disease in adults.

In our first study, we assessed carbon content in sputum macrophages from adult females and children exposed to biomass smoke [1]. As this study was conducted in a town (Gondar) where expensive equipment was not available, we first developed a simplified method to process the sputum sample. This new method, developed in Leicester, did not require the use of ice or a cytocentrifuge and, at the same time, did not affect the morphology of cells. The carbon content of AM in adult females and children exposed to biomass smoke was higher than in adults and children exposed to traffic-derived PM. The carbon content was lower in children compared with their mothers. This suggests that in children, a behaviour of staying away from fire helps to reduce exposure. 
In the second study, we investigated relationships between carbon in AM and: 1) exposure, 2) demographic variables, 3) lung function, and 4) exercise. There was a weak but positive association with annual mean modelled primary PM10 (particulate matter with a 50\% cut-off aerodynamic diameter of $10 \mu \mathrm{m})$ at a child's residence. The most significant finding was an inverse association of AM carbon content and lung function (forced expiratory volume in one second and mean forced expiratory flow between 25 and $75 \%$ of the forced vital capacity) [2]. There was no association of age, sex, body mass index or exercise with AM carbon content. AM carbon content in children with asthma was lower, despite living in higher PM areas, suggesting impaired uptake of PM.

In this large group of healthy children, we found that sputum neutrophil differential had a wide range, consistent with other studies. We investigated the role of oxidative stress and interleukin (IL)-8 [3]. This is the first study to measure 8-oxo7,8-dihydro-2'-deoxyguanosine (8-oxodG) in sputum supernatant. The induced sputum neutrophil percentage and absolute counts were associated with IL-8 but not with 8-oxodG measured in the induced sputum supernatant. We concluded that the variation in the proportion and number of neutrophils in the lower airway of healthy children is driven by IL-8.

\section{THE IMPACT OF MY WORK ON CLINICAL OR RESEARCH PRACTICE}

Our findings suggest that AM carbon can be used as a biomarker of individual lower airway PM exposure. This is probably a relevant marker with which to assess lower respiratory adverse health effects. AM had lower carbon content in children with asthma, suggesting a possible impaired phagocytosis of PM. This could be a mechanism of increased susceptibility to adverse effects of PM in subjects with asthma.

Health education to mothers using biomass fuels in developing countries should include simple measures, such as: 1) keeping children as far as possible from the fire, especially those aged $<5$ yrs; and 2) cooking either in well-ventilated rooms or in the open air to reduce exposure to high levels of particulate matter. Measurement of airway macrophage carbon content can be used to correlate exposure to adverse health effects, to strengthen the causal role of particulate matter and to study effectiveness of interventions, such as a smokeless stove.

\section{REFERENCES}

1 Kulkarni NS, Prudon B, Panditi SL, Abebe Y, Grigg J. Carbon loading of alveolar macrophages in adults and children exposed to biomass smoke particles. Sci Total Environ 2005; 345: 23-30.

2 Kulkarni N, Pierse N, Rushton L, Grigg J. Carbon in airway macrophages and lung function in children. $N$ Engl J Med 2006; 355: 21-30.

3 Kulkarni N, Cooke MS, Grigg J. Neutrophils in induced sputum from healthy children: role of interleukin-8 and oxidative stress. Respir Med 2007; 101: 2108-2112. 